\title{
Genome analysis of Limosilactobacillus fermentum JN2019 applied to tumeric fermentation for animal feed
}

\section{(4) Check for updates}

Received: May 13, 2021

Revised: Jun 2, 2021

Accepted: Jun 2, 2021

\#These authors contributed equally to this work

*Corresponding author

Sejong Oh

Division of Animal Science, Chonnam National University, Gwangju 61186,

Korea.

Tel: +82-62-530-2116

E-mail: soh@jnu.ac.kr

Copyright (C) 2021 Korean Society of Animal Sciences and Technology.

This is an Open Access article distributed under the terms of the Creative Commons Attribution

Non-Commercial License (http:// creativecommons.org/licenses/bync/4.0/) which permits unrestricted non-commercial use, distribution, and reproduction in any medium, provided the original work is properly cited.

ORCID

Heeseop Yoo

https://orcid.org/0000-0003-2905-6676

Cheng Chung Yong

https://orcid.org/0000-0003-0531-6381

Sejong Oh

https://orcid.org/0000-0002-5870-3038

Competing interests

No potential conflict of interest relevant to this article was reported.

Funding sources

This work was supported by the Basic Science Research Program through the National Research Foundation of Korea (NRF) funded by the Minister of Education, Science, and Technology (NRF-2019R1A2C108764811).

\author{
Heeseop Yoo ${ }^{1,2 \#}$, Cheng Chung Yong ${ }^{2 \#}$ and Sejong $\mathrm{Oh}^{2 *}$ \\ ${ }^{1} \mathrm{JNBIO}$, Jeongeup 56212, Korea \\ ${ }^{2}$ Division of Animal Science, Chonnam National University, Gwangju 61186, Korea
}

\begin{abstract}
Limosilactobacillus fermentum JN2019, formerly named Lactobacillus fermentum JN2019, was isolated from kimchi. Its genome was completely sequenced using the PacBio RSII sequencing system to explore beneficial phenotypes. In a previous study, L. fermentum JN2019 was used to ferment the by-product of tumeric for use in livestock feed. The $2.3 \mathrm{Mb}$ genome had a high guanine $(\mathrm{G})+$ cytosine $(\mathrm{C})$ content of $50.6 \%$ and a $30 \mathrm{~kb}$ plasmid. The data will inform the comprehensive understanding of JN2019 and provide insights for potential applications.

Keywords: Limosilactobacillus fermentum, Kimchi, Whole-genome sequencing, Feed, Lactobacillus
\end{abstract}

\section{ANNOUNCEMENT}

Limosilactobacillus fermentum (formerly named Lactobacillus fermentum JN2019) is commonly found in fermented food products and is generally considered safe [1]. L. fermentum has been regularly used for acid-producing starter cultures and acts as a food preservative [2]. In addition, JN2019 increases the bioavailability of curcumin, the active component of turmeric, while reducing cytotoxicity through fermentation [3]. In the present study, the JN2019 genome was sequenced to explore its genetic characteristics.

JN2019 was isolated from local fermented kimchi in Korea and grown in de Man-Rogosa-Sharpe (MRS) medium (Merck, Darmstadt, Germany). Genomic DNA (gDNA) was extracted with DNeasy Ultraclean microbial kit (Qiagen, Hilden, Germany), according to the manufacturer's instructions. The gDNA was sequenced using single molecular real-time (SMRT) portal (v.2.3) with the PacBio RS II system (Pacific Biosciences, Menlo Park, CA, USA). A total of 43,479,132 reads $(6,565,348,932$ total bases) were generated using SMRT sequencing. Gene neighborhood analysis illustrating the closest genome to JN2019 was L. fermentum strain DR9 (98.26\%), followed by strain FTDC 8312 (85.60\%). Although pronounced similarity to strain DR9 was observed, the value fell below the species recognition threshold of 98.6\% [4], clarifying the identity of JN2019 as L. fermentum. The genome sequences were annotated by the NCBI Prokaryotic Genomes Annotation Pipeline.

The complete genome of JN2019 consists of two contigs within the $2.3 \mathrm{Mb}$ genome $(\mathrm{G}+\mathrm{C}$ content 
Acknowledgements

Not applicable.

Availability of data and material Upon reasonable request, the datasets of this study can be available from the corresponding author.

Authors' contributions

Conceptualization: Oh S.

Data curation: Yoo $\mathrm{H}$, Yong CC.

Formal analysis: $\mathrm{Yoo} \mathrm{H}$, Yong $\mathrm{CC}$.

Methodology: $Y 00 \mathrm{H}$

Investigation: $\mathrm{Yoo} \mathrm{H}$.

Writing - original draft: Yong CC, Oh S.

Writing - review \& editing: Yong CC, Oh S.

Ethics approval and consent to participate This article does not require IRB/IACUC approval because there are no human and animal participants.
Table 1. Genome features of Limosilactobacillus fermentum JN2019

\begin{tabular}{lrc}
\hline & Chromosome & Plasmid \\
\hline Genome size (bp) & $2,298,221$ & 29,243 \\
G + C content (\%) & 50.6 & 40.8 \\
Gene & 2,359 & 34 \\
Pseudogene & 206 & 6 \\
Protein & 2,077 & 28 \\
rRNA & 15 & - \\
tRNA & 58 & - \\
Other RNA & 3 & - \\
\hline
\end{tabular}

G, guanine; C, cytosine.

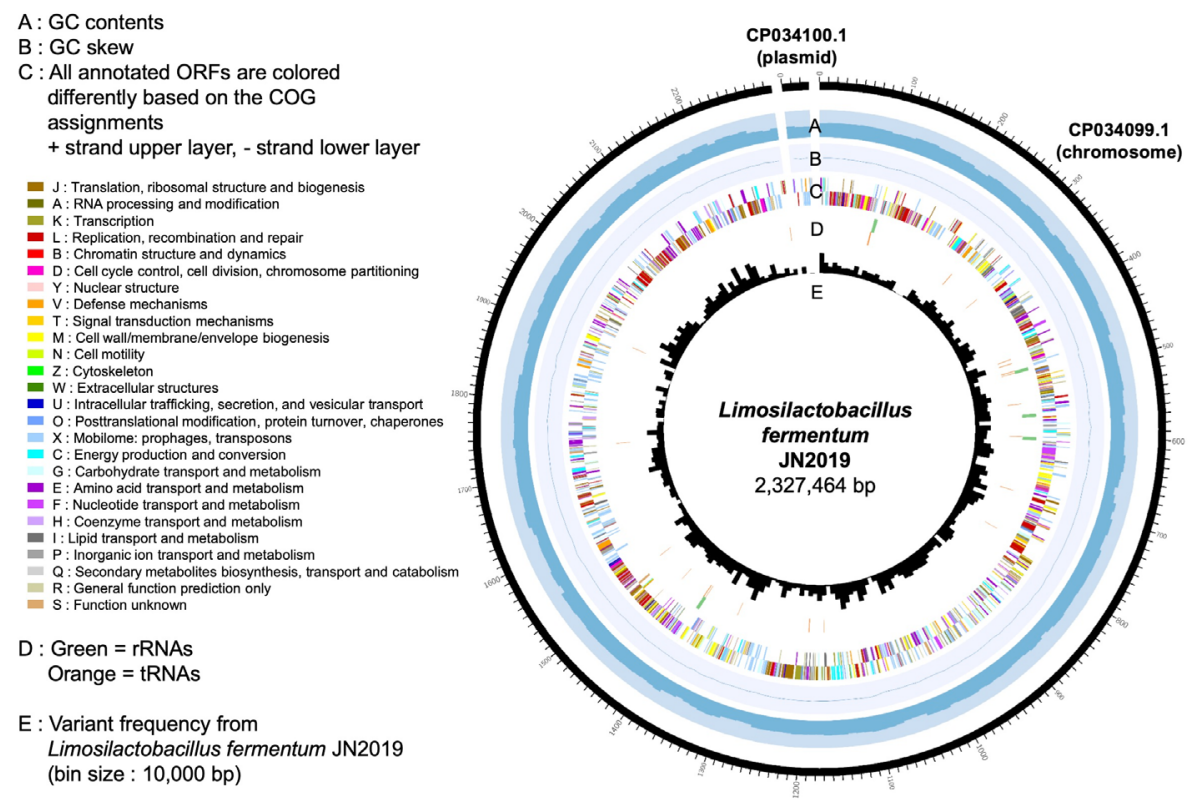

Fig. 1. Circular genome map of Limosilactobacillus fermentum JN2019. Circles from the outside to the center denote (A) G + C content, (B) G + C skew, (C) annotated open reading frames colored differently based on the COG assignments, (D) rRNAs (green) and tRNAs (orange), and (E) variant frequency. G, guanine; C, cytosine; ORF, open reading frame; COG, clusters of orthologous group.

of 50.5\%), a single chromosome of $2.3 \mathrm{Mb}$ with a $\mathrm{G}+\mathrm{C}$ content of $50.6 \%$ and a $30 \mathrm{~kb}$ plasmid with a $\mathrm{G}+\mathrm{C}$ content of $40.8 \%$ (Table 1). The $2.3 \mathrm{Mb}$ genome corresponds to 2,359 genes, 2,077 proteins, $15 \mathrm{rRNAs}, 58 \mathrm{tRNAs}$, and 3 other RNAs. These 2,359 genes are specifically clustered into 26 Clusters of Orthologous Groups of proteins-based functional categories (Fig. 1).

The genome information of JN2019 provides fundamental knowledge to inform discoveries of its beneficial properties and industrial applications. The complete genome sequence of JN2019 is available from NCBI/GenBank under BioSample accession number SAMN10417155 or directly via the assembly accession number CP034099.1 (chromosome) and CP034100.1 (plasmid).

\section{REFERENCES}

1. Zheng J, Wittouck S, Salvetti E, Franz CM, Harris HM, Mattarelli P et al. A taxonomic note 
on the genus Lactobacillus: description of 23 novel genera, emended description of the genus Lactobacillus Beijerinck 1901, and union of Lactobacillaceae and Leuconostocaceae. Int J Syst Evol Microbiol. 2020;70:2782-858. https://doi.org/10.1099/ijsem.0.004107

2. Swain MR, Anandharaj M, Ray RC, Parveen Rani R. Fermented fruits and vegetables of Asia: a potential source of probiotics. Biotechnol Res Int. 2014;2014:250424. https://doi. org/10.1155/2014/250424

3. Yong CC, Yoon Y, Yoo HS, Oh S. Effect of lactobacillus fermentation on the anti-inflammatory potential of turmeric. J Microbiol Biotechnol. 2019;29:1561-9. https://doi.org/10.4014/ jmb.1906.06032

4. Kim M, Oh HS, Park SC, Chun J. Towards a taxonomic coherence between average nucleotide identity and 16S rRNA gene sequence similarity for species demarcation of prokaryotes. Int J Syst Evol Microbiol. 2014;64:346-51. https://doi.org/10.1099/ijs.0.059774-0 